\title{
Thoracosternotomy for off-pump coronary artery bypass
}

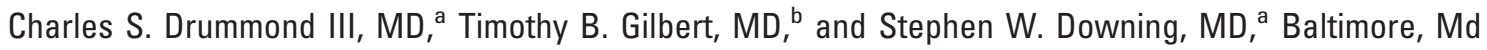

$\mathrm{T}$ he use of off-pump coronary artery bypass grafting (OPCAB) techniques may reduce risk, particularly for high-risk patients. ${ }^{1-2}$ However, many of the very patients who would benefit most from an OPCAB approach are the least tolerant of the requisite cardiac manipulation. We hypothesized that a left anterior thoracosternotomy (LATS) would reduce the amount of cardiac displacement (relative to a median sternotomy) required for exposure of lateral and inferior wall targets. This could facilitate OPCAB in patients with cardiomegaly and poor cardiac function that might render them relatively intolerant of conventional $\mathrm{OPCAB}$ techniques.

\section{Patients and Methods}

In 8 patients a LATS incision was used for OPCAB when an off-pump technique was indicated but the patient's cardiomegaly and severely reduced ejection fraction made exposure of the obtuse marginal vessels difficult or not technically feasible. The heart was approached by way of a LATS through the fourth or fifth intercostal space. The incision crossed the sternum and extended 4 to 5 $\mathrm{cm}$ into the right side of the chest (Figure 1). The left and right internal thoracic arteries (ITAs) were completely mobilized as indicated. The pericardium was opened laterally above the phrenic nerve. Traction on right-sided pericardial sutures provided adequate exposure of the ascending aorta for proximal anastomoses or cannulation. Heparin was administered to achieve an activated clotting time of 300 seconds. The Genzyme retractor-stabilizer (Genzyme Corporation, Cambridge, Mass) or the Octopus stabilizer (Medtronic, Inc, Minneapolis, Minn) was used for distal anastomoses.

\section{Results}

Mean ejection fraction was $24.4 \% \pm 13.1 \%$. Mean cardiothoracic ratio on chest radiography was $0.65 \pm 0.06$ ( $>50 \%$ indicates cardiomegaly). Two patients had intra-aortic balloon pumps in place before the operation for low cardiac output.

A total of 19 grafts were constructed in the 8 patients, with a mean of $2.4 \pm 0.5$ grafts per patient. Targets were left anterior descending coronary artery for 8 , diagonal artery for 2 , obtuse

\footnotetext{
From the Department of Surgery, Division of Cardiac Surgery, ${ }^{a}$ and the Departments of Anesthesiology and Medicine, ${ }^{\text {b }}$ University of Maryland School of Medicine, Baltimore, Md.

Received for publication May 19, 2002; accepted for publication May 31, 2002.

Address for reprints: Stephen W. Downing, MD, Cardiac Surgery, Suite N4W94, University of Maryland Medical System, 22 S Greene St, Baltimore, MD 21201 (E-mail: sdowning@smail.umaryland.edu).

J Thorac Cardiovasc Surg 2003;125:1157-8

Copyright $\odot 2003$ by The American Association for Thoracic Surgery

$0022-5223 / 2003 \$ 30.00+0$

doi: $10.1067 / \mathrm{mtc} .2003 .358$
}

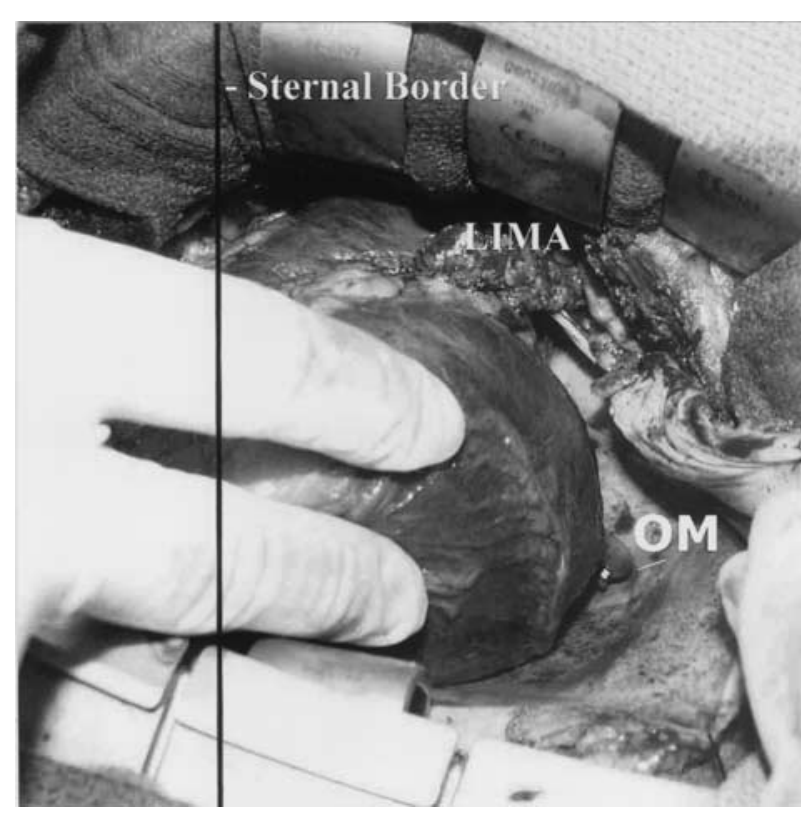

Figure 1. Surgeon's view of lateral wall via LATS. Black line marks left sternal borders. Graft to obtuse marginal branch (OM) is visible with minimal cardiac displacement. Left ITA graft (LIMA) to left anterior descending coronary artery can also be seen.

marginal vessels for 5 , ramus intermedius for 1 , right coronary artery for 1 , and posterior descending coronary artery for 2 . The conduits were as follows: left ITA, 7; right ITA, 2; saphenous vein, 7; and radial artery, 4 . Of 4 radial grafts, 3 originated from the right or left ITA. No additional inotropic agents (more than baseline) were required during surgery. The mean pulmonary artery pressure rose only by $16 \% \pm 7 \%$, and cardiac index declined an average of only $14 \% \pm 7 \%$ during distal anastomosis construction. The postoperative stay averaged $9.3 \pm 8.2$ days, with 4 patients discharged on day 4 . There were no deaths. There were no sternal complications noted in any patient at follow-up 4 weeks after the operation.

One patient required conversion to cardiopulmonary bypass because of difficulty dissecting out intramyocardial vessels. In that case, standard ascending aortic and right atrial cannulation and conventional cardioplegia were used.

\section{Comments}

This was a group of high-risk patients with uniformly reduced ejection fractions $(24.4 \% \pm 13.1 \%)$, cardiomegaly, and a high incidence of significant vascular disease (2 porcelain ascending aortas, 1 aortic occlusion, and 17 -cm suprarenal aortic aneurysm). This series represents a group of patients who would theoretically benefit from avoiding cardiopulmonary bypass and aortic cross- 
clamping. However, their cardiomegaly and reduced ejection fractions suggested difficulty in approaching targets on the lateral and inferior walls without hemodynamic compromise.

Several techniques have been developed to minimize the hemodynamic changes during $\mathrm{OPCAB}$, including volume loading, inotropes, patient positioning, deep pericardial retraction sutures, right pleurectomy, right hemisternal elevation, pulmonary artery shunts, intracoronary shunts, and cardiac assist devices. ${ }^{1-5}$ Even with full use of these techniques, however, exposure of lateral wall targets through a median sternotomy may not be possible for certain patients with severe cardiomegaly or poor function. The LATS incision was used in this group of patients because of the perception that it would allow the full range of surgical options for OPCAB, with less cardiac manipulation and hopefully less hemodynamic disruption than a midline incision. This is because, unlike a median sternotomy, a LATS incision is centered near the cardiac apex. Thus less rotation is needed to view the lateral and inferior walls, and there is no left sternal edge to obstruct the surgeon's view. In addition, the heart is not compressed against the sternum when rotated to the right.

The thoracosternal incision is associated with poor wound healing and an elevated risk of sternal nonunion. Also, rib-spreading incisions are notorious for causing postoperative pain. Fortunately, we did not observe any significant incision-related difficulties in our small series. However, more experience will be required before thoracosternotomy could be considered for broader application.

In conclusion, a LATS incision allows excellent exposure of all target vessels, with no significant compromises in routine operative technique. It should be considered in situations where multivessel OPCAB is indicated but the requisite cardiac displacement may be poorly tolerated.

\section{References}

1. Koutlas TC, Elbeery JR, Williams JM, Moran JF, Francalancia NA, Chitwood WR Jr. Myocardial revascularization in the elderly using beating heart coronary artery bypass surgery. Ann Thorac Surg. 2000; 69:1042-7.

2. Bergsland J, Hasnan S, Lewin AN, Bhayana J, Lojos TZ, Salerno TA. Coronary bypass grafting without cardiopulmonary bypass-an attractive alternative in high risk patients. Eur J Cardiothorac Surg. 1997; 11:876-80.

3. Mathison M, Edgerton JR, Horswell JL, Akin JJ, Mack MJ. Analysis of hemodynamic changes during beating heart surgical procedures. Ann Thorac Surg. 2000;70:1355-61.

4. Porat E, Sharony R, Ivry S, Ozaki S, Meyns BP, Flameng WJ, et al. Hemodynamic changes and right heart support during vertical displacement of the beating heart. Ann Thorac Surg. 2000;69:1188-91.

5. Luchetti V, Capasso F, Caputo M, Grimaldi G, Capece M, Brando G, et al. Intracoronary shunt prevents left ventricular function impairment during beating heart coronary revascularization. Eur J Cardiothorac Surg. 1999;15:255-9.

\section{Bilateral diaphragmatic paralysis after aortic surgery with topical hypothermia: Ventilatory assistance by means of nasal mask bilevel positive pressure}

Yoshiyuki Tokuda, MD, Masahiko Matsumoto, MD, Takaaki Sugita, MD, Junichiro Nishizawa, MD, Katsuhiko Matsuyama, MD, Kazunori Yoshida, MD, and Takehiko Matsuo, MD, Nara, Japan

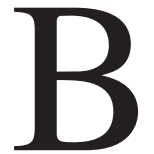

ilateral diaphragmatic paralysis is a rare but severe complication of cardiac surgery mainly caused by hypothermic injury of phrenic nerves. ${ }^{1,2}$ Most patients with bilateral diaphragmatic paralysis have been treated with positive-pressure ventilators through tracheostomy. ${ }^{3}$

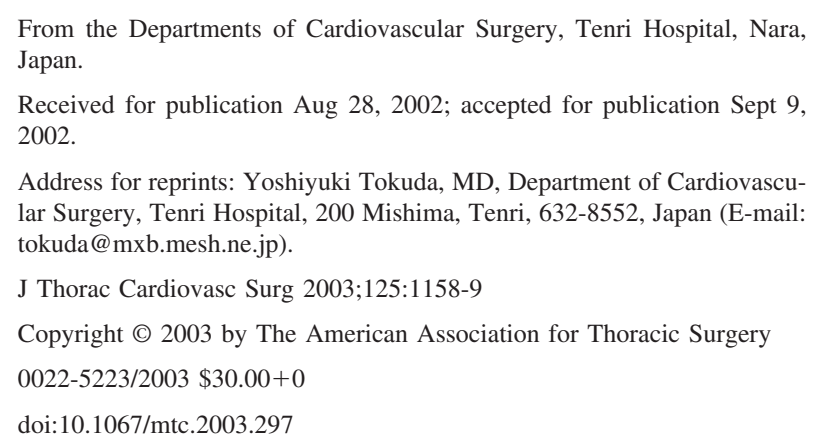

Recently, noninvasive positive airway ventilation has been applied to various types of respiratory failure. We report the case of a 35-year-old man who had postoperative bilateral diaphragmatic paralysis and was treated with nasal mask bilevel positive airway pressure (BiPAP) ventilation.

\section{Clinical Summary}

A 35-year-old man with a history of renal dysfunction and hypertension complained of sudden chest pain and was referred to our hospital. Computed tomography revealed the presence of Stanford type A acute aortic dissection and a large amount of pericardial effusion. The patient was in shock because of cardiac tamponade.

The patient underwent an emergency operation. During the operation, cardiopulmonary bypass was established with right atrial venous cannulation and left femoral arterial cannulation. The patient was cooled to $25^{\circ} \mathrm{C}$ by using the cardiopulmonary bypass circuit. The dilated ascending aorta was opened vertically, and antegrade selective cerebral perfusion was performed for all 3 arch vessels. For myocardial protection, St Thomas solution was di- 